\title{
电动势法测定硫酸铜的离子迁移数
}

孟德坤, 周南, 林世泉, 周爱秋, 林猛 ${ }^{*}$, 张树永

山东大学化学与化工学院, 济南 250100

摘要: 离子迁移数作为物理化学的重要参数, 其测定是大学物理化学实验中的基础内容。以基础物理化学实验中离 子迁移数的测定为基础, 结合电动势的测定等实验, 设计建立了一套电动势法测量溶液中铜离子与硫酸根离子迁移 数的实验装置, 得到的离子迁移数与文献值基本一致。尝试基于大学生在物理化学实验中已掌握的实验技能, 综合 电化学学科领域的知识, 设计探索电动势测量离子迁移数的新方法, 有助于提升学生对知识的综合运用能力。

关键词: 离子迁移数; 电动势法; 半透膜

中图分类号: G64; O64

\section{Determination of Ion Transference Number by Electromotive Forces Method}

Dekun Meng, Nan Zhou, Shiquan Lin, Aiqiu Zhou, Meng Lin *, Shuyong Zhang

School of Chemistry and Chemical Engineering, Shandong University, Jinan 250100, China.

Abstract: Ion transference number is an important parameter of physical chemistry, and its measurement is the basic content in physical chemistry laboratory. Based on the measurement of ion transference number in "Basic Physical Chemistry Laboratory", combined with measurements of the electromotive force, a new experiment was designed for measuring the transference number of copper ion and sulfate ion in solution, and the ion transference numbers obtained are basically consistent with the values in the literature. We attempt to use the laboratory skills that college students already mastered in physical chemistry laboratory and integrate the knowledge of electrochemistry to design and explore a new method for measuring ion transference by the electromotive force method. The students' comprehensive ability can be improved to solve problems.

Key Words: Ion transference number; Electromotive force method; Semi-permeable membrane

\section{1 引言}

“离子迁移数的测定” 是经典的物理化学教学实验。离子的迁移数可以通过实验直接获得, 常 用的测量方法有希托夫法、界面移动法和电动势法 ${ }^{[1,2]}$ 。希托夫法原理简单, 实验易于操作, 高校实 验多采用 “希托夫法测定离子迁移数” [3], 而在实验过程中希托夫法受对流、扩散、震动等影响, 易 出现测量结果不准确, 实验耗时久等缺点。界面移动法测量精度好, 但对许多离子较难获得清晰的 溶液界面, 操作要求高 ${ }^{[4]}$ 。电动势法具有快速获得较宽溶液浓度范围离子迁移数的特点。韩世钧及

收稿: 2021-01-05; 录用: 2021-01-26; 网络发表: 2021-02-22

”通讯作者, Email: mlin@sdu.edu.cn

基金资助：山东大学教育教学改革项目(2020Y229) 
其合作者 ${ }^{[5]}$ 通过改进Weingärtner等设计的电化学实验装置, 成功测定了溴化钾在醇水溶液中的离子 迁移数, 并讨论了迁移数在混合溶液中的变化规律。电动势法测定离子迁移数需要分别组装有溶液 接界和无溶液接界的浓差电池, 实验仪器要求较高, 而传统电池电动势的测定需要制备录齐化的可 逆电极, 录和亚录离子的使用不仅对师生的健康造成危害而且会造成环境污染 ${ }^{[6]}$, 因此, 利用电动 势法测定离子迁移数的相关讨论及实验设计相对较少。

在部分物理化学实验教材中对电动势法测定离子迁移数进行了简单的讨论 ${ }^{[3]}$ 。为了进一步拓展 实验教学内容, 提高学生对化学、物理等多学科领域知识的运用能力, 本文利用大学物理化学教学 实验中涉及的实验方法及测试手段, 设计了以半透膜分隔溶液, 铜棒为电极的浓差电池, 通过对 有/无液体接界的浓差电池电动势的测定, 计算得到硫酸铜水溶液中离子的迁移数, 以此增强学生对 离子迁移数的认识以及实验设计与探索的能力。由于铜棒在热加工过程中所产生的应力、应变等会 引起电动势的变化, 而且铜在空气中易发生氧化, 且很难保证所使用的铜棒组成完全一致 ${ }^{[7]}$ 。为消 除杂质及应力对电极电势的影响, 本实验采用电化学镀铜技术制备铜电极, 铜镀层的引入既可以保 证表面铜原子的活性, 使电极获得较大的比表面积, 具有较好的稳定性, 又能降低实验误差。该实 验还能避免剧毒录和亚录离子的使用 ${ }^{[8]}$ 。

\section{2 实验原理}

两种含有不同溶质或者两种相同溶质而浓度不同的溶液界面上存在的电势差被称为液接电 势 ${ }^{[9]}$ 。例如下列电池:

$\mathrm{Cu}\left|\mathrm{CuSO}_{4}\left(a_{1}\right)\right| \mathrm{CuSO}_{4}\left(a_{2}\right) \mid \mathrm{Cu}$

当电池输出 $1 \mathrm{~mol}$ 元电荷电量时, 则将有 $t_{+} / 2 \mathrm{~mol}$ 的 $\mathrm{Cu}^{2+}$ 从活度为 $\left(a_{\mathrm{Cu}^{2+}}\right)_{1}$ 的溶液通过界面迁移到 活度为 $\left(a_{\mathrm{Cu}^{2+}}\right)_{2}$ 的溶液, $t_{-} / 2 \mathrm{~mol}$ 的 $\mathrm{SO}_{4}^{2-}$ 从活度为 $\left(a_{\mathrm{SO}_{4}^{2-}}\right)_{2}$ 的溶液通过界面迁移到活度为 $\left(a_{\mathrm{SO}_{4}^{2-}}\right)_{1}$ 的溶液, 假定迁移数与溶液浓度无关, 则迁移过程吉布斯自由能变 $\Delta G_{\mathrm{j}}{ }^{[10]}$ :

$$
\Delta G_{\mathrm{j}}=\frac{t_{+}}{2} R T \ln \frac{\left(a_{\mathrm{Cu}^{2+}}\right)_{2}}{\left(a_{\mathrm{Cu}^{2+}}\right)_{1}}-\frac{t_{-}}{2} R T \ln \frac{\left(a_{\mathrm{SO}_{4}^{2-}}\right)_{2}}{\left(a_{\mathrm{SO}_{4}^{2-}}\right)_{1}}=-F E_{\mathrm{j}}
$$

其中 $t_{+}=1-t_{-}$, 则液接电势 $E_{\mathrm{j}}$ 为:

$$
E_{\mathrm{j}}=\left(t_{-}-t_{+}\right) \frac{R T}{2 F} \ln \frac{\left(a_{\mathrm{Cu}^{2+}}\right)_{2}}{\left(a_{\mathrm{Cu}^{2+}}\right)_{1}}=\left(2 t_{-}-1\right) \frac{R T}{2 F} \ln \frac{\left(a_{\mathrm{Cu}^{2+}}\right)_{2}}{\left(a_{\mathrm{Cu}^{2+}}\right)_{1}}
$$

具有液体接界电势的电池的电动势, 可由公式 $E=E_{0}+E_{\mathrm{j}}$ 求得 (其中 $E$ 为实测电动势, $E_{0}$ 为无溶液 接界的电池电动势, $E_{\mathrm{j}}$ 为液接电势)。

而溶液接界可以忽略的浓差电池 $\mathrm{Cu}\left|\mathrm{CuSO}_{4}\left(a_{1}\right)_{\| !} \mathrm{CuSO}_{4}\left(a_{2}\right)\right| \mathrm{Cu}$, 其电动势 ${ }^{2}{ }^{[11]}$ :

$$
E_{0}=\frac{R T}{2 F} \ln \frac{\left(a_{\mathrm{Cu}^{2+}}\right)_{2}}{\left(a_{\mathrm{Cu}^{2+}}\right)_{1}}
$$

则有溶液接界的浓差电池 $\mathrm{Cu}\left|\mathrm{CuSO}_{4}\left(a_{1}\right)\right| \mathrm{CuSO}_{4}\left(a_{2}\right) \mid \mathrm{Cu}$, 其电动势为:

$$
E_{1}=E_{0}+E_{\mathrm{j}}=\frac{R T}{2 F} \ln \frac{\left(a_{\mathrm{Cu}^{2+}}\right)_{2}}{\left(a_{\mathrm{Cu}^{2+}}\right)_{1}}+\left(2 t_{\mathrm{SO}_{4}^{2^{-}}}-1\right) \frac{R T}{2 F} \ln \frac{\left(a_{\mathrm{Cu}^{2+}}\right)_{2}}{\left(a_{\mathrm{Cu}^{2+}}\right)_{1}}=2 t_{\mathrm{SO}_{4}^{2-}} \frac{R T}{2 F} \ln \frac{\left(a_{\mathrm{Cu}^{2+}}\right)_{2}}{\left(a_{\mathrm{Cu}^{2+}}\right)_{1}}
$$

由式(3)和(4)可知, 离子迁移数可通过有/无液体接界浓差电池的电动势之比来求算, 即:

$$
\frac{E_{1}}{E_{0}}=\frac{2 t_{\mathrm{SO}_{4}^{2-}} \frac{R T}{2 F} \ln \frac{\left(a_{\mathrm{Cu}^{2+}}\right)_{2}}{\left(a_{\mathrm{Cu}^{2+}}\right)_{1}}}{\frac{R T}{2 F} \ln \frac{\left(a_{\mathrm{Cu}^{2+}}\right)_{2}}{\left(a_{\mathrm{Cu}^{2+}}\right)_{1}}}=2 t_{\mathrm{SO}_{4}^{--}}
$$

因此 $t_{\mathrm{SO}_{4}^{2-}}=\frac{1}{2} \frac{E_{1}}{E_{0}}, t_{\mathrm{Cu}^{2+}}=1-t_{\mathrm{SO}_{4}^{2-}}$ 。 


\section{3 试剂及仪器}

火棉胶溶液, 硫酸铜溶液, 饱和氯化钾溶液, 滤纸条, 铜棒两根(99.9\%), 滤纸, $100 \mathrm{~mL}$ 容量瓶, $25 \mathrm{~mL}$ 移液管, $250 \mathrm{~mL}$ 锥形瓶, 烧杯若干, 铁架台, 胶头滴管, U型玻璃管, 数显恒温水浴槽(HH-1), 电镀装置(倍思特直流稳压电源PS-1502DD, 电流表, 滑动变阻器), EM-3C数字式电位差计(南京大 万科教)。

\section{4 实验步骤}

\section{1 制备半透膜}

火棉胶半透膜的制备参见文献 ${ }^{[3]}$ 。将火棉胶膜取出后, 放入盛有 $0.10 \mathrm{~mol} \cdot \mathrm{L}^{-1}$ 硫酸铜溶液的烧杯 中备用。

\section{2 电镀电极}

铜棒用砂纸打磨至露出金属光泽后, 用蒸馏水洗净。铜电镀液每升含 $125 \mathrm{~g}$ 五水硫酸铜, $25 \mathrm{~g}$ 硫 酸和 $50 \mathrm{~mL}$ 乙醇, 电镀电压为 $6 \mathrm{~V}$, 电流为 $20 \mathrm{~mA}$, 时间为 $30 \mathrm{~min}$ 。铜棒做阴极, 纯铜板做阳极。电镀 得到的铜电极, 洗净后放入 $0.10 \mathrm{~mol} \cdot \mathrm{L}^{-1}$ 硫酸铜溶液中备用。

\section{3 简易盐桥的制备}

U型玻璃管用去离子水洗净后, 用饱和氯化钾溶液润洗。随后将饱和氯化钾溶液小心注入U型玻 璃管中, 用滤纸条塞入U型管的两端, 制备好盐桥备用 ${ }^{[12]}$ 。

\section{4 配制硫酸铜溶液}

准确称取 $25.00 \mathrm{~g}$ 五水硫酸铜, 用去离子水配制成 $0.10 \mathrm{~mol} \cdot \mathrm{L}^{-1}$ 的硫酸铜储备溶液。采用稀释的方 法，分别配制 $0.01 、 0.02 、 0.025 、 0.04 、 0.05 、 0.06 \mathrm{~mol} \cdot \mathrm{L}^{-1}$ 的硫酸铜溶液备用。

\section{5 数字电位差计的使用}

\subsection{1 校准}

数字电位差计的红黑导线分别接入外标的正负极, 测量开关拨至 “外标”, 导线短接, 待稳定后, 按下 “调零” 校准。随后将导线接入外标 “标准电动势”, 调节旋钮使 “电动势指示” 与仪器上的标 准电动势相同, 按下 “调零” 进行校准。

\subsection{2 测量}

校准后将开关拨至 “测量”, 导线插入电位差计测量电路的正负极, 并接入待测电池, 待示数稳 定后, 调节旋钮, 使 “平衡指示” 为零, 此时仪器 “电动势指示” 显示的电动势数值即为该电池的 电动势。

\section{6 实验装置}

利用电动势法测定硫酸铜的离子迁移数实验装置如图1、图2所示, 图1为测定有液接电势浓差电 池的电动势实验装置, 图2为测定无液接电势浓差电池的电动势实验装置。

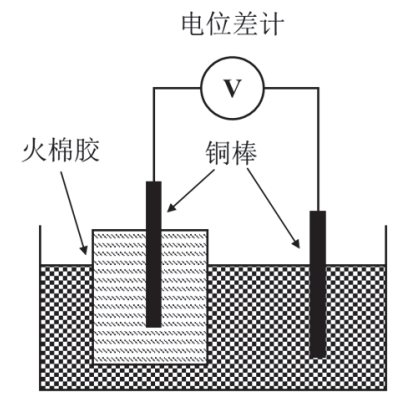

图1＼cjkstart测定有液接电势浓差电池的电动势实验装置示意图

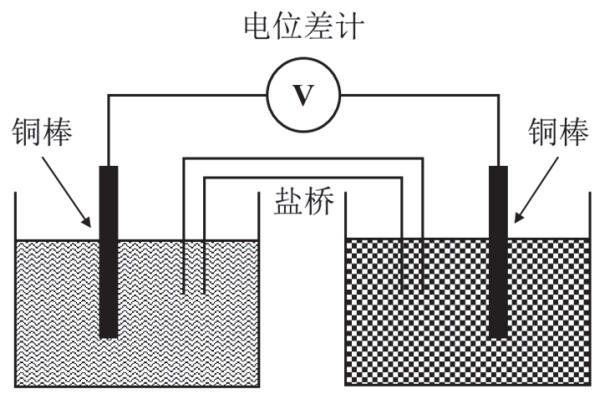

图2 测定无液接电势浓差电池的电动势实验装置示意图 


\section{7 实验装置的组装与电动势的测量}

将制备好的半透膜取出, 用蒸馏水洗净后, 依次加入 $10 \mathrm{~mL} 0.01 \mathrm{~mol} \cdot \mathrm{L}^{-1}, 0.02 \mathrm{~mol} \cdot \mathrm{L}^{-1}, 0.04 \mathrm{~mol} \cdot \mathrm{L}^{-1}$, $0.05 \mathrm{~mol} \cdot \mathrm{L}^{-1}, 0.06 \mathrm{~mol} \cdot \mathrm{L}^{-1}$ 的硫酸铜溶液 (该浓度设为 $c_{1}$ ), 并将其置于盛有 $30 \mathrm{~mL} 0.10 \mathrm{~mol} \cdot \mathrm{L}^{-1}$ (浓度设 为 $c_{2}$ ) 的硫酸铜溶液的烧杯中。电镀好的铜棒用蒸馏水冲洗后分别放入烧杯和半透膜中。放入半透膜 中的铜棒接负极, 烧杯中的铜棒接正极, 即为有液接电势的浓差电池(图1)。同时, 另取两个干燥洁净 的 $50 \mathrm{~mL}$ 烧杯, 分别加入 $30 \mathrm{~mL}$ 浓度为 $0.10 \mathrm{~mol} \cdot \mathrm{L}^{-1}\left(c_{2}\right)$ 的硫酸铜溶液和 $10 \mathrm{~mL}$ 浓度为 $c_{1}\left(0.01 \mathrm{~mol} \cdot \mathrm{L}^{-1}\right.$, $\left.0.02 \mathrm{~mol} \cdot \mathrm{L}^{-1}, 0.04 \mathrm{~mol} \cdot \mathrm{L}^{-1}, 0.05 \mathrm{~mol} \cdot \mathrm{L}^{-1}, 0.06 \mathrm{~mol} \cdot \mathrm{L}^{-1}\right)$ 的硫酸铜溶液, 放入盐桥连接, 插入铜棒。 $10 \mathrm{~mL}$ 溶液中的铜棒接接负极, $30 \mathrm{~mL}$ 溶液中的铜棒接正极, 即为无液接电势的浓差电池(图2)。将上 述组装好的电池放入 $25 \pm 0.2{ }^{\circ} \mathrm{C}$ 恒温水浴中恒温 $5 \mathrm{~min}$ 后, 利用数字电位差计分别测量两个电池的 电动势, 重复三次并取平均值。随后, 将浓度为 $c_{2}$ 的 $0.10 \mathrm{~mol} \cdot \mathrm{L}^{-1}$ 硫酸铜溶液替换为 $0.05 \mathrm{~mol} \cdot \mathrm{L}^{-1}$ 的 硫酸铜溶液, 在半透膜中分别加入 $0.01 、 0.02 、 0.025 、 0.04 \mathrm{~mol} \cdot \mathrm{L}^{-1}\left(c_{1}\right)$ 的硫酸铜溶液, 重复上述实 验步骤, 测量两个电池的电动势, 重复三次并取平均值。

\section{5 数据处理与讨论}

利用公式 $t_{\mathrm{SO}_{4}^{2-}}=E_{1} / 2 E_{0}, t_{\mathrm{Cu}^{2+}}=1-t_{\mathrm{SO}_{4}^{2-}}$, 由表 1 计算可得, 当正极溶液为 $0.10 \mathrm{~mol} \cdot \mathrm{L}^{-1}$ 硫酸铜溶液时, 平均的离子迁移数: $t_{\mathrm{SO}_{4}^{2-}}=0.552, t_{\mathrm{Cu}^{2+}}=0.448$ 。当正极溶液为 $0.05 \mathrm{~mol} \cdot \mathrm{L}^{-1}$ 硫酸铜溶液时, 平均的离 子迁移数： $t_{\mathrm{SO}_{4}^{2-}}=0.558, t_{\mathrm{Cu}^{2+}}=0.442$ 。

查表可知 ${ }^{[10,11]}, 25^{\circ} \mathrm{C}$ 水溶液中 $1 / 2 \mathrm{Cu}^{2+}$ 的极限摩尔电导率 $\lambda_{+}^{\infty}=54.0 \mathrm{~S} \cdot \mathrm{cm}^{2} \cdot \mathrm{mol}^{-1}, 25^{\circ} \mathrm{C}$ 水溶液中 $1 / 2 \mathrm{SO}_{4}^{2-}$ 的极限摩尔电导率 $\lambda_{-}^{\infty}=79.8 \mathrm{~S} \cdot \mathrm{cm}^{2} \cdot \mathrm{mol}^{-1}$ 。由标准数据计算得离子迁移数为 ${ }^{[13]}$ :

$$
\begin{aligned}
& t_{\mathrm{SO}_{4-}^{2-}}=\lambda_{-}^{\infty} /\left(\lambda_{+}^{\infty}+\lambda_{-}^{\infty}\right)=79.8 /(54.0+79.8)=0.596 \\
& t_{\mathrm{Cu}^{2+}}=\lambda_{+}^{\infty} /\left(\lambda_{+}^{\infty}+\lambda_{-}^{\infty}\right)=54.0 /(54.0+79.8)=0.404
\end{aligned}
$$

离子迁移数的理论值是基于无限稀释的数据进行计算的, 而实际测量时因离子间存在作用力, 应该用活度替代浓度。利用电动势法测量离子迁移数, 其计算公式仅涉及到浓差电池的电动势, 故 活度未在文中讨论。本实验利用电动势法测得硫酸根的离子迁移数 $t_{\mathrm{SO}_{4}^{2-}}$ 比理论值稍低, 但相差较小 (偏差 $7.4 \%$ ), 而在体系溶液浓度较低时, 计算得到的离子迁移数更接近理论值。其原因为实验选用 的硫酸铜体系中二价离子间的吸引力较大, 当溶液浓度改变时, 离子间的静电引力等发生较大变化, 从而对浓差电池的电动势产生影响, 使所测得的电动势偏离理论值。

\begin{tabular}{|c|c|c|c|c|c|}
\hline $\begin{array}{l}\text { 硫酸铜浓度 } \\
c_{2} /\left(\mathrm{mol} \cdot \mathrm{L}^{-1}\right)\end{array}$ & $\begin{array}{l}\text { 硫酸铜浓度 } \\
c_{1} /\left(\mathrm{mol} \cdot \mathrm{L}^{-1}\right)\end{array}$ & $\begin{array}{c}\text { 无液接电池电动势 } \\
E_{0} / \mathrm{mV}\end{array}$ & $\begin{array}{c}\text { 有液接电池电动势 } \\
E_{1} / \mathrm{mV}\end{array}$ & $t \mathrm{so}_{4}^{2-}$ & $t \mathrm{Cu}^{2+}$ \\
\hline \multirow[t]{5}{*}{0.10} & 0.01 & 18.47 & 18.61 & 0.504 & 0.496 \\
\hline & 0.02 & 12.85 & 14.85 & 0.577 & 0.423 \\
\hline & 0.04 & 8.15 & 9.11 & 0.559 & 0.441 \\
\hline & 0.05 & 6.0 & 6.42 & 0.535 & 0.465 \\
\hline & 0.06 & 4.46 & 5.26 & 0.589 & 0.411 \\
\hline \multirow[t]{4}{*}{0.05} & 0.01 & 16.23 & 17.03 & 0.525 & 0.475 \\
\hline & 0.02 & 7.92 & 9.15 & 0.578 & 0.422 \\
\hline & 0.025 & 6.12 & 6.83 & 0.558 & 0.442 \\
\hline & 0.04 & 3.73 & 4.26 & 0.571 & 0.429 \\
\hline
\end{tabular}

表 1 数字电位差计测得的不同浓差电池的电动势 $\left(25.0 \pm 0.2{ }^{\circ} \mathrm{C}\right)$ 


\section{6 结语}

本文通过对电动势测量离子迁移数的思考, 利用大学物理化学实验课程中所涉及半透膜制备、 电动势的测定及离子迁移数的测定等实验的原理、操作及仪器, 有机整合了大学物理化学基础实验 内容, 设计了浓差电池及电动势测量装置, 成功实现了电动势法测定硫酸铜溶液中离子迁移数的目 的。该法较希托夫法和界面移动法实验装置更简便、易操作, 测量速度快、准确率高, 能够满足大 学物理化学实验教学要求。本实验从具体问题出发, 综合学生所掌握的知识点, 通过对所提问题进 行分析、讨论, 有助于培养学生化学实验的素养和能力。

\section{参 考 文 献}

[1] 张春芳, 霍丙南, 韩琳玉, 姚宁, 沈福刚, 商艳丽, 孙素芳, 单金缓, 马志广. 化学教育, 2020, 41 (14), 96.

[2] 詹世景, 吴植宇, 李林杰, 陈秀娟, 王静, 李细雄, 范枫枫, 吕京美. 广州华工, 2017, 45 (8), 149

[3] 宋淑娥. 基础化学实验(III)——物理化学实验. 第3版. 北京: 化学工业出版社, 2020: 145.

[4] 张国林, 刘正铭. 实验室研究与探索, 2000, No. 4, 100.

[5] 姚加, 闰卫东, 谢学鹏, 韩世钧. 浙江大学学报, 1999, 33 (1), 33 .

[6] 墽锦晴, 薛树忠, 耿文英. 大学化学, 1994, 9 (1), 35 .

[7] 陈耕野, 唐理想, 田军, 李风起, 潘智. 沈阳建筑大学学报(自然科学版), 2006, 22 (6), 925 .

[8] 李苍, 张虎成, 张树霞, 代冬梅. 大学化学, 2014, 29 (2), 59.

[9] 王清华. 科技资讯, 2009, No. 1, 34.

[10] 傅献彩, 沈文霞, 姚天扬, 侯文华. 物理化学. 第5版. 北京: 高等教育出版社, 2018: 21-75.

[11] 印永嘉, 奚正楷, 张树永. 物理化学简明教程. 第4版. 北京: 高等教育出版社, 2018: 223-258.

[12] 顾月姝. 物理化学实验. 济南: 山东大学出版社, 2002: 142.

[13] 胡扬剑, 欧阳跃军. 怀化学院学报, 2004, 23 (2), 37. 